\title{
A morphological study of the medial and lateral femoral circumflex arteries: a proposed new classification
}

\author{
P. Łabętowicz' ${ }^{1}$, Ł. Olewnik' ${ }^{1}$ M. Podgórski², M. Majos³ ${ }^{3}$ L. Stefańczyk ${ }^{3}$, M. Topol ${ }^{4}$, M. Polguj \\ ${ }^{1}$ Department of Normal and Clinical Anatomy, Chair of Anatomy and Histology, Medical University of Lodz, Poland \\ 2Department of Diagnostic Imaging, Polish Mother's Memorial Hospital Research Institute, Lodz, Poland \\ ${ }^{3}$ Department of Radiology, Barlicki University Hospital, Medical University of Lodz, Poland \\ ${ }^{4}$ Department of Angiology, Chair of Anatomy and Histology, Medical University of Lodz, Poland
}

[Received: 12 December 2018; Accepted: 28 January 2019]

Background: Confirming the branching pattern of the deep femoral artery (DFA) is vital in planning radiological and surgical procedures involving the medial circumflex femoral artery (MFCA) and the lateral circumflex artery (LFCA). The aim of this study was to characterise the course and morphology of branches of the DFA. Materials and methods: The anatomical dissection included 80 lower limbs which were fixed in 10\% formalin solution. A dissection of the femoral region was carried out according to a pre-established protocol, using traditional techniques. Morphometric measurements were obtained twice by two researchers.

Results: Six types of medial and lateral femoral circumflex artery variations were distinguished. In type I, the DFA divides into the MFCA and the LFCA (observed in $45 \%$ of cases). In type II, the MFCA is absent and the LFCA origin normally from the DFA (18.75\%). In type III, the MFCA arises from the femoral artery above the origin of the DFA, while the LFCA starts from the DFA (15\%). Finally, in type IV, the LFCA arises from the femoral artery above the origin of the DFA, while the MFCA starts from the DFA (10\%). In type $V$, the LFCA origin alone from the femoral artery below the origin of the DFA, while the MFCA origin from the DFA (7.5\%), while in type VI (3.75\%), both the MFCA and the LFCA origin from the femoral artery. The mean diameter of the femoral artery at the level of the DFA origin was greatest in type $2(10.62 \pm 2.07 \mathrm{~mm})$ and the least in type 6 (7.90 \pm $\pm 1.72 \mathrm{~mm} ; p=0.0317)$. The distance from inguinal ligament to where the DFA arose was the greatest in type $6(78.24 \pm 29.74 \mathrm{~mm})$ and least in type 5 (28.85 $\pm 11.72 \mathrm{~mm} ; p=0.0529)$.

Conclusions: The medial and lateral femoral circumflex arteries were characterised by high morphological variations. The diameter of the femoral artery at the level of inguinal ligament correlated with the diameter of the DFA and distance to where the DFA arises from femoral artery. (Folia Morphol 2019; 78, 4: 738-745)

Key words: anatomy, classification, deep femoral artery, lateral circumflex femoral artery, medial circumflex femoral artery, variations

Address for correspondence: Assoc. Prof. M. Polguj, Department of Normal and Clinical Anatomy, Chair of Anatomy and Histology, Medical University of Łódź, ul. Żeligowskiego 7/9, 90-752 Łódź, Poland, e-mail: michal.polguj@umed.lodz.pl 


\section{INTRODUCTION}

The anatomical variations of the vessels of the lower limb have long received attention from various authors $[3,5-9,12-14,19-22]$. Within the arterial network of the lower limb, a number of variations can be seen regarding the morphology of the femoral artery (FA) and its main branches, especially the medial femoral circumflex artery (MFCA) and lateral femoral circumflex artery (LFCA) $[8,9,14,19,22$, 24-26]. The FA is found below the inguinal ligament as a continuation of the external iliac artery, and serves as the main vessel carrying blood to the lower limb. The main branch of the FA is the deep femoral artery (DFA), which provides blood for the entire thigh area, including the hip joint, femur and the anterior, medial and posterior groups of muscles [25]. The LFCA and the MFCA are typically the largest branches of the DFA; however, the LFCA is topographically posterolateral, while the MFCA is posteromedial [25].

Surgical and radiological procedures in the area of the hip joint are frequent, so knowledge of possible anatomical variants of the FA and its branches is essential in everyday practice. For example, orthopaedists performing total hip replacements, trochanteric or intratrochanteric osteotomies $[14,24]$ and radiologist performing transluminal stent implementation and angioplasty, embolectomy or diagnostic angiography $[28,29]$ will find such information valuable.

Despite the large number of studies performed on the variations of the MFCA and the LFCA, knowledge of the area remains insufficient. The aim of our study is therefore to identify variations of the medial and lateral circumflex femoral artery which may be relevant for minimising complications after diagnostic tests and surgical/orthopaedic procedures in the thigh region. In addition, we propose a new classification that takes into account both the LFCA and the MFCA together; we believe this approach to be more accurate than that given in most previous publications as these only consider individual variations.

\section{MATERIALS AND METHODS}

The anatomical dissection included 80 lower limbs (34 female and 46 male) which were fixed in 10\% formalin solution. None of the specimens demonstrated any trace of surgical intervention in the lower leg region. The protocol of the study was accepted by the Bioethics Committee of the Medical University of Lodz (resolution no. RNN/10/18/KE). A dissection of the femoral region was carried out according to a pre-established protocol, using traditional techniques [16-18].

The thigh region was approached and the fat was removed. The femoral nerve and femoral vein were identified and separated from the FA. During the dissection, the morphology of FA and DFA were evaluated, particularly its branching pattern in the region of the femoral triangle. Next, the external diameter of the FA (at the inguinal ligament, below and before the point where the DFA arises), DFA (at the FA), MFCA (at the DFA or FA) and LFCA (at the DFA or FA) was evaluated. Also, the distance of the point where the DFA arises from FA was measured. All morphometric measurements were obtained with an electronic calliper (Mitutoyo Corporation, Kawasaki-shi, Kanagawa, Japan). Each measurement was taken twice by two researchers with considerable experience in anatomical dissection, and was accurate to within $0.1 \mathrm{~mm}$. In further calculations, the mean value of four measurements was used.

All measurements of arteries at the femoral region were carried out according to a previously described protocol using traditional techniques [16-18].

\section{Statistical analysis}

We performed the statistical analysis using Statistica 12 software (StatSoft Polska, Krakow, Poland). A p-value lower than 0.05 was considered significant. The results are presented as mean and standard deviation unless otherwise stated.

The Student's t-test was used to assess the association between sexes/body sides and types of arterial branching patterns. The normality of the distribution of continuous data was checked with the Shapiro-Wilk test. As the data was not normally distributed, the Mann-Whitney test and the Wilocoxon sign-rank test were used to compare anthropometric measurements between sexes and body sides, respectively. The correlation between measurements was checked with the Spearman's rank correlation test. The Kruskal-Wallis ANOVA with dedicated post hoc test was used to compare these measurements between types of arterial tree. The Bonferroni correction was applied for multiple testing.

\section{RESULTS}

Based on the obtained anatomical variations of the main branches of the DFA, six basic types were distinguished, some of which also include subtypes. - type I - both the MFCA and LFCA departed from the DFA in $36(45 \%)$ specimens. Based on point 

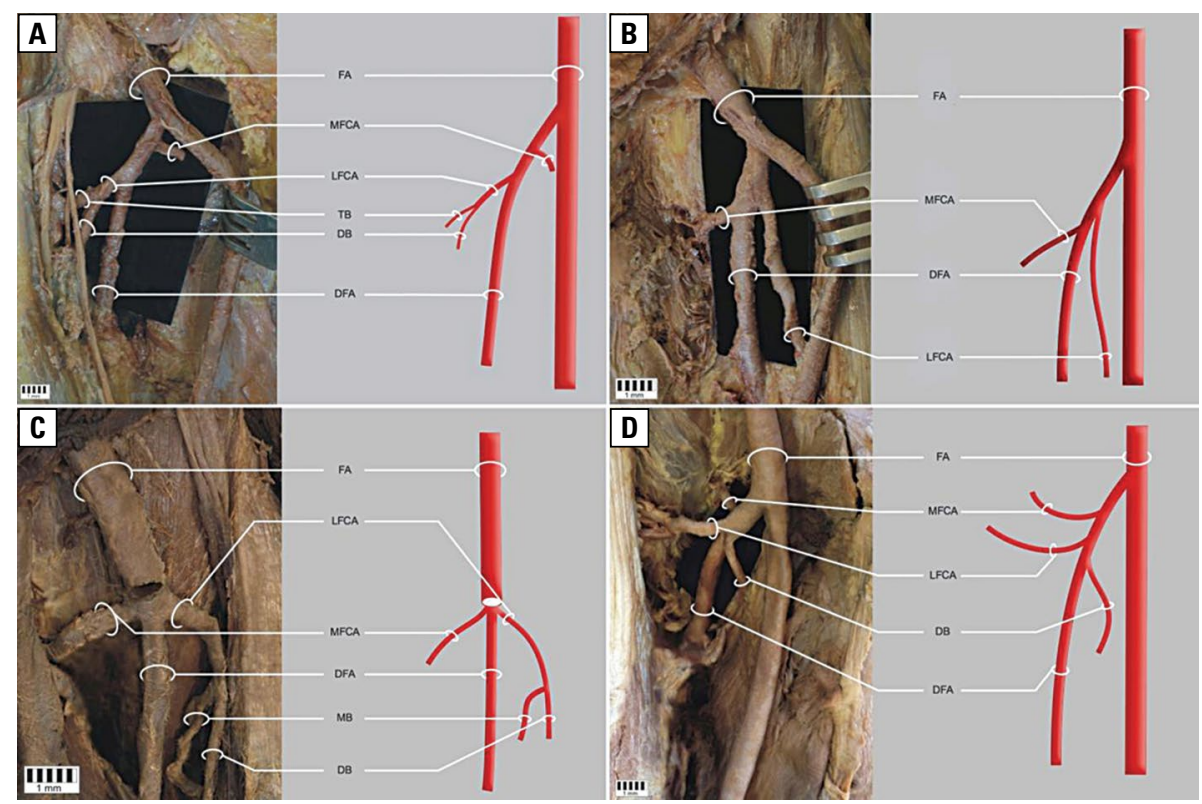

Figure 1. Type 1 medial and lateral femoral circumflex artery variation; A. Subtype 1a; B. Subtype 1b; C. Subtype 1c; D. Subtype 1d; FA — femoral artery; MFCA — medial femoral circumflex artery; LFCA — lateral femoral circumflex artery; DFA — deep femoral artery; $\mathrm{TB}$ - transverse branch; $\mathrm{DB}$ — descending branch; $\mathrm{MB}$ — muscular branch.

where the branches arose, this type was divided into four subtypes:

- la - the MFCA origin above the LFCA, observed in 27 of all specimens (33.75\%) (Fig. 1A),

- $\mathrm{lb}$ - the LFCA origin above the MFCA, observed in five of all specimens (6.25\%) (Fig. 1B),

- Ic - the MFCA and the LFCA go down at the same altitude, observed in two lower limbs (2.5\%) (Fig. 1C),

- Id - the MFCA origin above the LFCA, while the descending branch (DB) also starts from the DFA below them (it usually leaves as a branch of the LFCA), observed in two of all specimens (2.5\%) (Fig. 1D);

- type II - the MFCA is absent and the LFCA origin as normal from the DFA, observed in 15 of all specimens (18.75\%) (Fig. 2);

- type III - the MFCA arises from the FA above the departure of DFA, while the LFCA origin from the DFA, observed in 12 of all specimens (15.0\%) (Fig. 3);

- type IV - the LFCA arises from the FA above the origin of the DFA, while the MFCA origin from the DFA, observed in 8 specimens $(10.00 \%)$ (Fig. 4);

- type $\mathrm{V}$ - the LFCA origin alone from the FA below the origin of the DFA, while the MFCA origin from the DFA. This type occurred in $6(7.5 \%)$ of all cases and was divided into two subtypes $(a, b)$ :

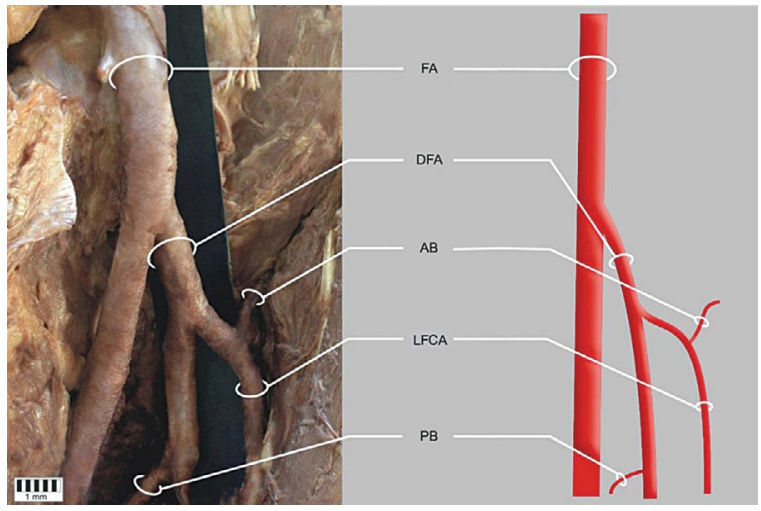

Figure 2. Type 2 medial and lateral femoral circumflex artery variation; FA — femoral artery; DFA - deep femoral artery; $A B$ - ascending artery; LFCA — lateral femoral circumflex artery; $\mathrm{PB}$ - perforating branch.

- $\mathrm{Va}$ - the LFCA origin from the FA below the origin of FA, while the MFCA origin as normal from the DFA, observed in 5 of all cases (6.25\%) (Fig. 5A),

- $\mathrm{Vb}$ - the DFA origin with a common trunk with the superficial circumflex iliac artery whereas the LFCA and the MFCA origin in the same way as type $\mathrm{Va}$, observed in only $1(1.25 \%)$ case, i.e. on 1 lower limb (Fig. 5B);

- type $\mathrm{VI}$ - both the MFCA and the LFCA origin from the DFA, this type occurred in 3 of all cases 


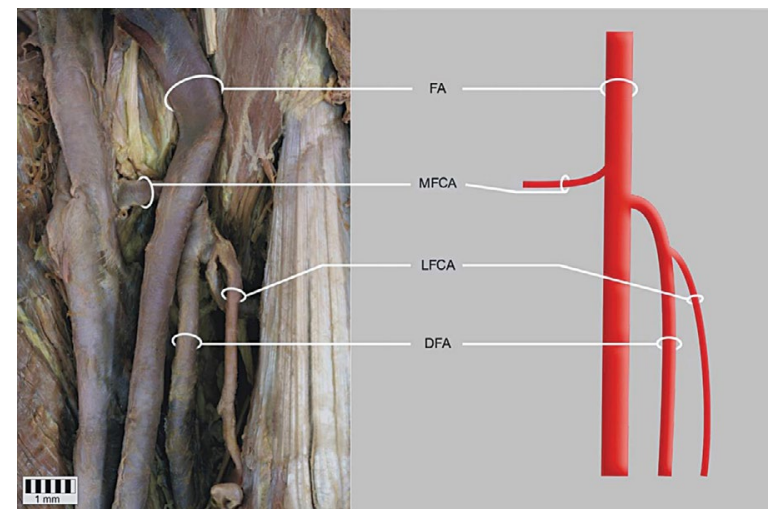

Figure 3. Type 3 medial and lateral femoral circumflex artery variation; FA - femoral artery; MFCA - medial femoral circumflex artery; LFCA — lateral femoral circumflex artery; DFA — deep femoral artery.
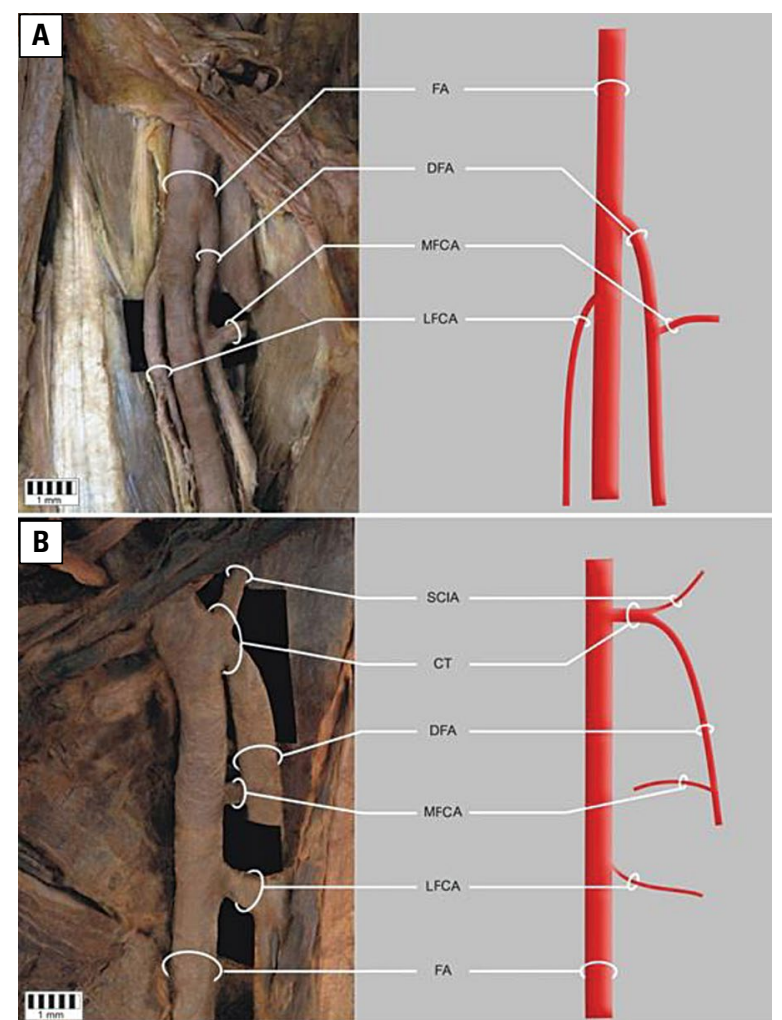

Figure 5. Type 5 medial and lateral femoral circumflex artery variation; A. Subtype 5a; B. Subtype 5b; FA — femoral artery; MFCA — medial femoral circumflex artery; LFCA — lateral femoral circumflex artery; DFA - deep femoral artery; CT — common trunk; SCIA — superficial circumflex iliac artery.

(3.75\%) and was also divided into two subtypes $(\mathrm{a}, \mathrm{b})$ :

- Vla - the MFCA and LFCA origin from FA above the origin of the DFA, with the MFCA above the LFCA, occurred in two of all cases (2.5\%) (Fig. 6A),

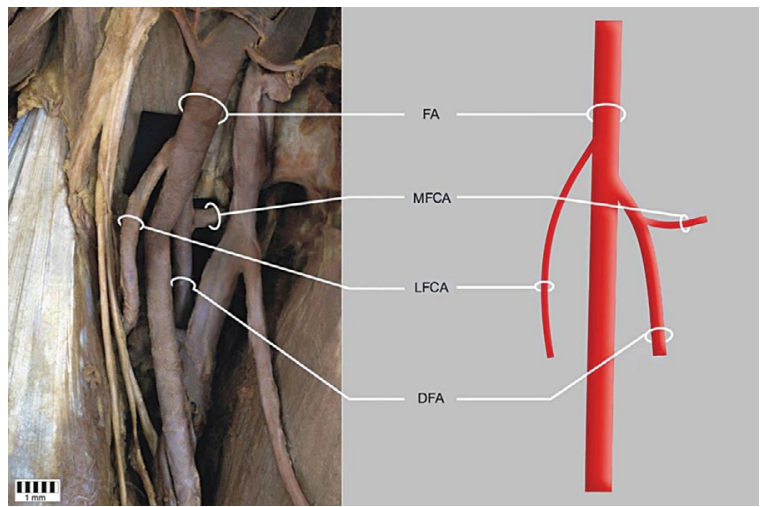

Figure 4. Type 4 medial and lateral femoral circumflex artery variation; FA — femoral artery; MFCA - medial femoral circumflex artery; LFCA — lateral femoral circumflex artery; DFA — deep femoral artery.
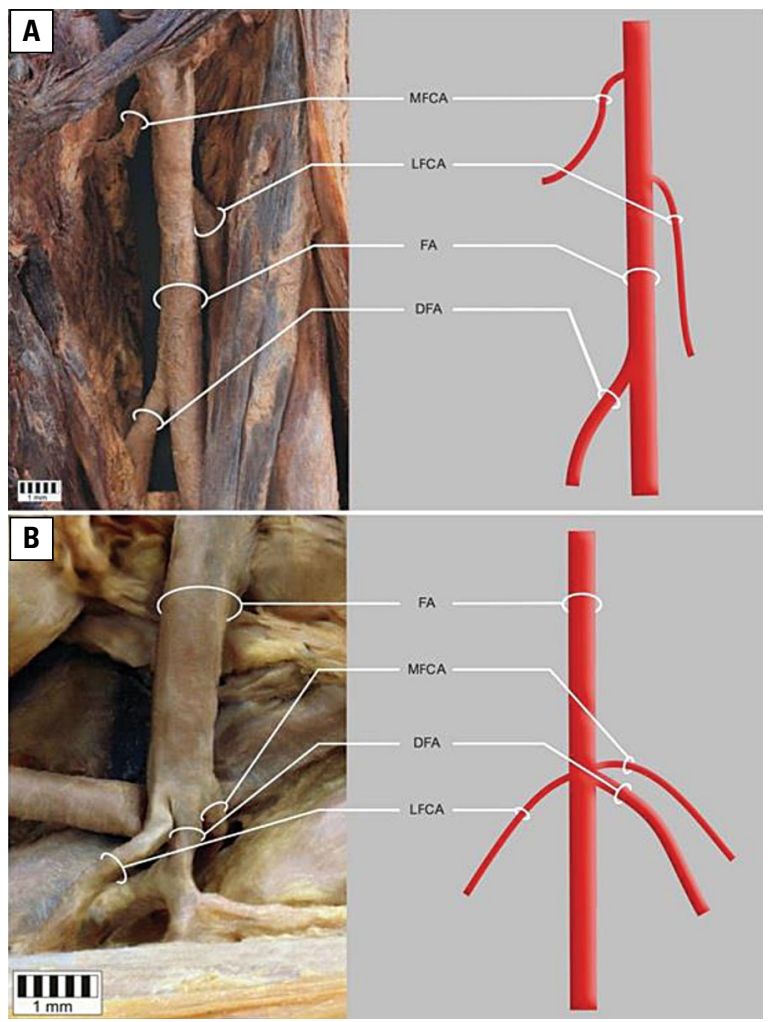

Figure 6. Type 6 medial and lateral femoral circumflex artery variation; A. Subtype 6a; B. Subtype 6b; FA - femoral artery; MFCA — medial femoral circumflex artery; LFCA — lateral femoral circumflex artery; DFA - deep femoral artery.

- $\quad \mathrm{VIb}$ - the MFCA and LFCA origin from the FA at the same altitude as the DFA, observed in only 1 (1.25\%) case, on one lower limb (Fig. 6B).

Morphometric analysis of the whole group found that a greater diameter of the FA at the level of the inguinal ligament correlated with a greater distance 
Table 1. Diameter of arteries with regard to gender

\begin{tabular}{lccc}
\hline Parameter [mm] & Female & Male & $\mathbf{P}$ \\
\hline FA diameter (inguinal ligament) & $9.94 \pm 1.65$ & $12.11 \pm 1.24$ & 0.0000 \\
FA diameter (before DFA) & $9.08 \pm 1.74$ & $10.91 \pm 1.49$ & 0.0000 \\
FA diameter (at DFA) & $8.50 \pm 1.89$ & $10.25 \pm 1.82$ & 0.0001 \\
FA diameter (below DFA) & $6.35 \pm 1.32$ & $8.23 \pm 1.42$ & 0.0000 \\
DFA diameter (start) & $6.42 \pm 1.39$ & $7.11 \pm 1.31$ & 0.0248 \\
MFCA diameter (start) & $3.56 \pm 1.04$ & $4.25 \pm 1.01$ & 0.0079 \\
LFCA diameter (start) & $4.74 \pm 1.10$ & $5.46 \pm 1.21$ & 0.0084 \\
\hline
\end{tabular}

FA — femoral artery; DFA — deep femoral artery; LFCA — lateral circumflex femoral artery; MFCA - medial circumflex femoral artery

to where the DFA arose $\left(R^{2}=0.29 ; p=0.02\right)$. Moreover, the diameter of the FA at the level of the inguinal ligament correlated significantly and positively with the diameters of the DFA $\left(R^{2}=0.25 ; p=0.0001\right)$, MCFA $\left(R^{2}=0.26 ; p=0.0001\right)$ and LCFA $\left(R^{2}=0.17\right.$; $\mathrm{p}=0.0005)$.

The diameters of all arteries were significantly greater in men than in women (Table 1).

Only one statistically significant trend was observed between the proposed taxa of medial and lateral femoral circumflex artery variations (Table 2; bolded): the mean diameter of the FA at the level of the DFA origin was greatest in type 2 (10.62 \pm $\pm 2.07 \mathrm{~mm})$ and least in type $6(7.90 \pm 1.72 \mathrm{~mm}$; $\mathrm{p}=0.0317)$. However, it is worth noting that a statistically insignificant difference was found regarding the distance between the inguinal ligament and the point where the DFA arises; this distance was greatest in type $6(78.24 \pm 29.74 \mathrm{~mm})$ and least in type 5 $(28.85 \pm 11.72 \mathrm{~mm} ; \mathrm{p}=0.0529)$.

Other insignificant relationships between performed measurements and the main types of branching pattern are presented in Table 2.

\section{DISCUSSION}

In order to understand arterial variations of the FA, it is necessary to understand the arterial embryology of the lower extremities. In foetal life, the sciatic artery, a branch of the internal iliac artery, is initially responsible for the vascularisation of the entire lower limb; however, when the foetus is eight weeks old and $10 \mathrm{~mm}$ long, it regresses and this function is taken over by the FA, a branch of the external iliac artery [19]. During early development, anastomoses develop between the dorsal sciatic artery and ventral FA [10].

At the same time, capillaries in front of the pelvis and thigh form complex vascular networks named the rete pelvicum and rete femorale, respectively [4], which increases the flow of blood in the capillaries and allows the arterial network to mature. Thus, the most appropriate channels enlarge whilst others contract and disappear, thereby establishing the final arterial pattern $[23,28]$. It is now recognised that anomalies that affect the arteries of the limbs are based on an ambiguous selection of channels from the primary capillary plexus associated with an increase in capillary pressure $[11,28]$.

The literature describes a number of variations in the mean distance between the midpoint of the inguinal ligament and the origin of the DFA. The origin of the DFA from the FA is usually stated to be about $4 \mathrm{~cm}$ distance from the inguinal ligament [4]; however, Snell reports its mean distance to be $4 \mathrm{~cm}$, Sidharth $4.4 \mathrm{~cm}$, and Dixit $4.75 \mathrm{~cm}$ [23]. A similar value was noted in the present study $43.15 \mathrm{~mm}$. On the other hand, Manjappa and Prasanna [11] report a mean distance of $3.56 \mathrm{~cm}$ for the right hand limb and $3.195 \mathrm{~cm}$ for the left.

Several surgical procedures require precise knowledge of the distance of the origin of the DFA from FA.

Table 2. Measurements of the medial and lateral femoral circumflex arteries according to the proposed classification

\begin{tabular}{lccccccc}
\hline Parameter [mm] & Type 1 & Type 2 & Type 3 & Type 4 & Type 5 & Type 6 & P \\
\hline DFA inguinal ligament & $43.14 \pm 12.85$ & $36.66 \pm 18.77$ & $48.33 \pm 14.77$ & $42.34 \pm 15.50$ & $28.85 \pm 11.72$ & $78.24 \pm 29.74$ & 0.0529 \\
FA d. (inguinal ligament) & $10.71 \pm 2.09$ & $11.42 \pm 1.57$ & $11.39 \pm 1.75$ & $12.07 \pm 1.08$ & $11.61 \pm 0.62$ & $11.77 \pm 0.83$ & 0.2673 \\
FA d. (before DFA) & $9.89 \pm 2.05$ & $10.56 \pm 1.71$ & $10.17 \pm 1.72$ & $10.74 \pm 1.76$ & $10.20 \pm 1.20$ & $9.02 \pm 1.50$ & 0.3895 \\
FA d. (at DFA) & $\mathbf{8 . 9 8} \pm \mathbf{2 . 1 6}$ & $\mathbf{1 0 . 6 2} \pm \mathbf{2 . 0 7}$ & $\mathbf{9 . 6 9} \pm 1.59$ & $\mathbf{1 0 . 0 8} \pm \mathbf{1 . 7 7}$ & $\mathbf{9 . 5 6} \pm \mathbf{1 . 2 5}$ & $\mathbf{7 . 9 0} \pm \mathbf{1 . 7 2}$ & $\mathbf{0 . 0 3 1 7}$ \\
FA d. (below DFA) & $7.26 \pm 1.93$ & $7.44 \pm 1.46$ & $7.49 \pm 1.33$ & $7.87 \pm 1.64$ & $8.02 \pm 0.92$ & $6.90 \pm 2.19$ & 0.8301 \\
DFA d. (start) & $6.81 \pm 1.31$ & $6.53 \pm 1.19$ & $6.51 \pm 1.22$ & $7.70 \pm 1.75$ & $7.51 \pm 1.17$ & $5.89 \pm 2.44$ & 0.3248 \\
MFCA d. (start) & $3.70 \pm 1.14$ & $4.42 \pm 0.73$ & $4.05 \pm 0.98$ & $4.60 \pm 0.86$ & $4.47 \pm 0.89$ & $3.46 \pm 0.99$ & 0.0775 \\
LFCA d. (start) & $4.99 \pm 1.39$ & $5.24 \pm 0.95$ & $5.44 \pm 0.84$ & $5.71 \pm 1.23$ & $5.10 \pm 1.18$ & $4.19 \pm 1.25$ & 0.1789 \\
\hline
\end{tabular}

FA — femoral artery; DFA — deep femoral artery; LFCA — lateral circumflex femoral artery; MFCA — medial circumflex femoral artery; d. — diameter 
Table 3. Comparison of the origins of the medial circumflex femoral artery by different studies

\begin{tabular}{lcccc}
\hline Author & Types of examination & Number of lower limbs - total & From DFA (directly) & From FA (directly) \\
\hline Our study & Anatomical dissection & 80 & $50(62.50 \%)$ & $15(18.75 \%)$ \\
Vazquez et al. (2007) & Anatomical dissection & 438 & $366(83.56 \%)$ & $72(16.4 \%)$ \\
Vuksanović-Božarić et al. (2018) & Anatomical dissection & 60 & $47(78.3 \%)$ & $3(5.0 \%)$ \\
Zlotorowicz et al. (2018) & CT - angiograms & 100 & $18(18 \%)$ & $34(34.0 \%)$ \\
Al.-Talalwah (2015) & Anatomical dissection & 342 & $195(57.0 \%)$ & $188(54.9 \%)$ \\
Tanyeli et al. (2006) & Anatomical dissection & 100 & $79(79 \%)$ & $15(15.0 \%)$ \\
Prakash et al. (2010) & Anatomical dissection & 64 & $43(67.2 \%)$ & $21(32.8)$ \\
Gautier et al. (2000) & Anatomical dissection & 24 & $20(83.3 \%)$ & $4(16.7 \%)$ \\
Siddharth et al. (1985) & Anatomical dissection & 100 & $63(63.0 \%)$ & $37(37.0 \%)$ \\
Nasr et al. (2013) & Anatomical dissection & 90 & $53(58.9 \%)$ & $37(41.1)$ \\
Adachi (1928) & Anatomical & 367 & $67.2 \%$ & $14 \%$ \\
Lippert and Pabst (1985) & Anatomical & 100 & $58 \%$ & $18 \%$ \\
\hline
\end{tabular}

CT — computed tomography; FA — femoral artery; DFA — deep femoral artery

It should be remembered that high artery origin can cause great difficulties when performing femoral arterial and venous puncture or femoral nerve blocks. However, our findings indicate that a greater FA diameter at the level of the inguinal ligament correlates with a greater distance of the point of origin of the DFA.

In our study, the most common type of occurrence of the LFCA and the MFCA was when they origin from the DFA, which was observed in $36(45 \%)$ lower limbs. Regarding the place of origin of the MFCA, the most common points of origin observed in the present study were from the DFA $(62.5 \%)$ and from the FA (18.75\%). Adachi reports that the MFCA originated from the DFA in $67.2 \%$ of cases and from the FA in $14 \%$ [1]. Another study conducted by Lippert and Pabst [10] also found the MFCA to originate most often from the DFA (58\%) and to a lesser extent from the FA (18\%). Similar observations have been made by Vazquez et al. [28], Vuksanovic-Bozaric et al. [29], Tanyeli et al. [24], Prakash et al. [20], Gautier et al. [8], Siddharth et al. [22], and Nasr et al. [14]. In contrast, Zlotorowicz et al. [30] found them to origin more often from the FA than from the DFA, and a study by Al-Talalwah [2] found the frequencies of occurrence to be quite similar, i.e. the MFCA originated from the DFA in $57 \%$, and from the FA in $54.9 \%$. A meta-analysis by Tomaszewski et al. [25] of 38 studies found that the MFCA originated from the DFA in $64.6 \%$ in cases, and from the FA in $32.2 \%$. A detailed comparison with other studies is presented in Table 3.
Regarding the lateral side, our present findings indicate that the LFCA originated more frequently from the DFA (78.85\%) than the FA (21.25\%). Adachi [1] observed that the LFCA most commonly originated from the DFA (78.2\%), with the second most common origin being the FA (18.3\%). Lippert and Pabst [10] found that the LFCA originated from the DFA in $76 \%$ of cases, and from the FA in 19\%. Similar observations have been made by Uzuel et al. [27], Vuksanovic-Bozaric et al. [29], Zlotorowicz et al. [30], Fakuda et al. [6], and Prakash et al. [20]. In the meta-analysis conducted by Tomaszewski et al. [25], the prevalence pooled from 26 studies found the LFCA to originate from the DFA in $76.61 \%$ of cases and from the FA in $19.1 \%$. A detailed comparison with other studies is presented in Table 4.

Medial circumflex femoral artery aplasia is quite rare, i.e. with a frequency below $1 \%$ being identified in previous studies [19]. In the present study, this frequency was found to be $18.75 \%$. A comparison of the frequency of MFCA aplasia between studies is presented in Table 5.

There are several different classifications in the literature of the variability of the LFCA and the MCFA, but most do not the combined approach of the origin of these vessels [2, 24-26].

In 2007, Vazquez et al. [28] proposed a new threefold classification of the branches of the FA: type I, with three subtypes, type II, with two subtypes, and type III, with no subtypes. The meta-analysis by Tomaszewski et al. [25, 26], proposed two new fivefold classifications, one of the LFCA and another of the MFCA, that also considered different subtypes. 
Table 4. Comparison of origin of the lateral circumflex femoral artery in different studies

\begin{tabular}{lcccc}
\hline Author & Types of examination & Number of lower limbs - total & From DFA (directly) & From FA (directly) \\
\hline Our study & Anatomical dissection & 80 & $63(78.75 \%)$ & $17(21.25 \%)$ \\
Uzel et al. (2008) & Anatomical dissection & 110 & $85(77.3 \%)$ & $21(19.1 \%)$ \\
Vazquez et al. (2007) & Anatomical dissection & 438 & $416(94.98 \%)$ & $22(5.02 \%)$ \\
Vuksanović-Božarić et al. (2018) & Anatomical dissection & 60 & $50(83.3 \%)$ & $1(1.7 \%)$ \\
Zlotorowicz et al. (2018) & CT - angiograms & 100 & $81(81.0 \%)$ & $65(65 \%)$ \\
Fukuda et al. (2005) & Angiography & 262 & $214(81.68 \%)$ & $45(17.17 \%)$ \\
Prakash et al. (2010) & Anatomical dissection & 64 & $52(81.25 \%)$ & $12(18.75 \%)$ \\
Adachi (1928) & Anatomical dissection & 367 & $78.2 \%$ & $18.3 \%$ \\
Lippert and Pabst (1985) & Anatomical dissection & & $76 \%$ & $18.3 \%$ \\
\hline
\end{tabular}

CT — computed tomography; FA — femoral artery; DFA — deep femoral artery

Table 5. Comparison of aplasia of the medial circumflex femoral artery

\begin{tabular}{lcccc}
\hline Author & Types of examination & Number of lower limbs - total & Per cent & No. of lower limbs \\
\hline Vuksanović-Božarić et al. (2018) & Anatomical dissection & 60 & 1.7 & 1 \\
Vazquez et al. (2007) & Anatomical dissection & 438 & 0 & 0 \\
Zlotorowicz et al. (2018) & CT - angiograms & 100 & 1 & 1 \\
Al-Talalwah (2015) & Anatomical dissection & 342 & 0.6 & 2 \\
Tanyeli et al. (2006) & Anatomical dissection & 100 & 0 & 0 \\
Prakash et al. (2010) & Anatomical dissection & 64 & 0 & 0 \\
\hline
\end{tabular}

CT — computed tomography

Vuksanovic-Bozaric et al. [29] proposed a new classification for foetuses based on the observed frequency of variation of MFCA and LFCA departures in 30 foetuses. Both Vazquez et al. [28] and Vuksanovic-Bozaric et al. [29] found the LFCA and MFCA to origin most often from the DFA. A 2018 computed tomography angiogram study of the anatomical variability of the MFCA and the LFCA found the most common form, i.e. occurring in $50 \%$ of cases, to be the trunks profundocircumflexus perfectus, in which the MFCA and the LFCA origin from the DFA [30], with the trunk profundocircumflexus lateralis, where the LFCA origin directly from the DFA, observed in $31 \%$ of cases and the trunk profundocircumflexus medialis, where the MFCA origin directly from the DFA, observed in 15\%.

In contrast, our proposed classification is a proprietary classification which more accurately classifies MFCAs and LFCAs, treating them in combination rather than separately, as in most previous publications. It is also complementary to classifications Vazquez et al. [28], Tomaszewski et al. [25, 26] and Zlotorowicz et al. [30].
Knowledge of the variability associated with the MFCA and LFCA origin is significant clinically. The branches of the FA supply the head and neck of the femur, and iatrogenic or traumatic damage to these vessels or to the hip joint itself can affect the ischaemia of the joint and result in its necrosis [12].

In the case of orthopaedic surgery, for example when treating hip joint fractures, knowledge of the arterial supply of the hip is extremely important [9]. Anastomoses between the arterial branches of the MFCA and the branches of the inferior gluteal artery are also important considerations in the case of hip dislocation [30]. An understanding of the variability of origin of the LFCA is valuable when using it for aortopopliteal bypass in cases of thrombosis of the iliofemoral arteries and the anterolateral thigh flaps [24] or in coronary artery bypass grafting [15]; these procedures can incorporate the descending branch derived from the LFCA [24]. In addition, the anatomical variations of the arteries of the thigh are important considerations when performing endovascular treatments from the FA [15]. Our classification reflects the variability of both the MFCA and the LFCA. It also 
takes into account the order of origin of the MFCA and the LFCA.

\section{CONCLUSIONS}

The medial and lateral femoral circumflex arteries were characterised by a high degree of morphological variation. The most common variant of the branch of the DFA was the presence of a departure by the LFCA. A wider diameter of the FA at the level of inguinal ligament correlated with a wider diameter of the DFA and greater distance to where the DFA arose from FA. Only the mean diameter of the FA at the level of the DFA origin showed any significant variation with regard to classification type, being highest in type 2 and lowest in type 6.

\section{Acknowledgements}

The authors wish to express their gratitude to all those who donated their bodies to medical science. The paper was supported by statutory research activity no. 503/2-031-01/503-21-004-17.

\section{REFERENCES}

1. Adachi B. Das Arteriensystem der Japaner. Verlag der Kais Univ zu Kyoto. 1928; 2: 18-71.

2. Al-Talalwah $\mathbf{W}$. The medial circumflex femoral artery origin variability and its radiological and surgical intervention significance. Springerplus. 2015; 4: 149, doi: 10.1186/s40064-015-0881-2, indexed in Pubmed: 25883882.

3. Aslaner R, Pekcevik $Y$, Sahin $H$, et al. Variations in the origin of inferior phrenic arteries and their relationship to celiac axis variations on CT angiography. Korean J Radiol. 2017; 18(2): 336-344, doi: 10.3348/kjr.2017.18.2.336, indexed in Pubmed: 28246513.

4. Bergman RA, Afifi AK, Miyauchi R. Anatomy Atlases: Illustrated Encyclopedia of Human Anatomic Variation - Anatomical Variation | Radiology Anatomy | Anatomy Atlas. 2015.

5. Chomiak J, Horák M, Masek M, et al. Computed tomographic angiography in proximal femoral focal deficiency. J Bone Joint Surg Am. 2009; 91(8): 1954-1964, doi: 10.2106/JBJS.H.00902, indexed in Pubmed: 19651955.

6. Fukuda $H$, Ashida $M$, Ishii $R$, et al. Anatomical variants of the lateral femoral circumflex artery: an angiographic study. Surg Radiol Anat. 2005; 27(3): 260-264, doi: 10.1007/s00276-004-0312-5, indexed in Pubmed: 15682274.

7. Freitas A, Aires HN, Pansiere ST, et al. Anatomical description of the proximal third of the medial femoral circumflex artery. A cadaveric study. Acta Ortop Bras Brazilian Soc Orthop Traumatol. 2012; 20: 21-24.

8. Gautier E, Ganz K, Krügel N, et al. Anatomy of the medial femoral circumflex artery and its surgical implications. J Bone Joint Surg Br. 2000; 82(5): 679-683, indexed in Pubmed: 10963165.

9. Kaempf M, Ketelsen D, Syha R, et al. CT angiography of various superficial femoral artery stents: an in vitro phantom study. Eur J Radiol. 2012; 81(7): 1584-1588, doi: 10.1016/j.ejrad.2011.04.014, indexed in Pubmed: 21546182.

10. Lippert $H$, Pabst R. Arterial variations in man. Classification and frequency. 1985, doi: 10.1007/978-3-642-80508-0_1.

11. Manjappa T, Prasanna LC. Anatomical variations of the profunda femoris artery and its branches: a cadaveric study in South Indian population. Indian J Surg. 2014; 76(4): 288-292, doi: 10.1007/ s12262-012-0677-3, indexed in Pubmed: 25278652.
12. Massoud TF, Fletcher EW. Anatomical variants of the profunda femoris artery: an angiographic study. Surg Radiol Anat. 1997; 19(2): 99-103, indexed in Pubmed: 9210243.

13. Mauro MA, Jaques PF, Moore M. The popliteal artery and its branches: embryologic basis of normal and variant anatomy. AJR Am J Roentgenol. 1988; 150(2): 435-437, doi: 10.2214/ ajr.150.2.435, indexed in Pubmed: 3257338.

14. Nasr AY, Badawoud MH, Al-Hayani AA, et al. Origin of profunda femoris artery and its circumflex femoral branches: anatomical variations and clinical significance. Folia Morphol. 2014; 73(1): 58-67, doi: 10.5603/FM.2014.0008, indexed in Pubmed: 24590524.

15. Olasińska-Wiśniewska A, Grygier M, Lesiak M, et al. Femoral artery anatomy-tailored approach in transcatheter aortic valve implantation. Post Kardiol Int. 2017; 13(2): 150-156, doi: 10.5114/ pwki.2017.68050, indexed in Pubmed: 28798786.

16. Olewnik $\measuredangle$, Waśniewska $A$, Polguj $M$, et al. Rare combined variations of renal, suprarenal, phrenic and accessory hepatic arteries. Surg Radiol Anat. 2018; 40(7): 743-748, doi: 10.1007/s00276-0182026-0, indexed in Pubmed: 29667030.

17. Olewnik $\measuredangle$, Wysiadecki G, Polguj $M$, et al. A rare anastomosis between the common hepatic artery and the superior mesenteric artery: a case report. Surg Radiol Anat. 2017; 39(10): 1175-1179, doi: 10.1007/s00276-017-1859-2, indexed in Pubmed: 28432408.

18. Olewnik $Ł$, Wysiadecki G, Polguj M, et al. Types of coeliac trunk branching including accessory hepatic arteries: a new point of view based on cadaveric study. Folia Morphol. 2017; 76: 660-667, doi: 10.5603/FM.a2017.0053, indexed in Pubmed: 28612916

19. Perera J. Anatomy of the origin of the deep femoral artery. Ceylon Med J. 1995; 40(4): 139-141, indexed in Pubmed: 8689700.

20. Prakash, Kumari J, Kumar Bhardwaj A, et al. Variations in the origins of the profunda femoris, medial and lateral femoral circumflex arteries: a cadaver study in the Indian population. Rom J Morphol Embryol. 2010; 51(1): 167-170, indexed in Pubmed: 20191139.

21. Rajani SJ, Ravat MK, Rajani JK, et al. Cadaveric Study of Profunda Femoris Artery with Some Unique Variations. J Clin Diagn Res. 2015; 9(5): AC01-AC03, doi: 10.7860/JCDR/2015/13913.5876, indexed in Pubmed: 26155466.

22. Siddharth $P$, Smith NL, Mason RA, et al. Variational anatomy of the deep femoral artery. Anat Rec. 1985; 212(2): 206-209, doi: 10.1002/ar.1092120216, indexed in Pubmed: 3842043.

23. Sinnatamby C. Last's anatomy: regional and applied. Churchill Livingstone Elsevier, Toronto 2006.

24. Tanyeli E, Uzel M, Yildirim M, et al. An anatomical study of the origins of the medial circumflex femoral artery in the Turkish population. Folia Morphol. 2006; 65(3): 209-212, indexed in Pubmed: 16988917.

25. Tomaszewski KA, Henry BM, Vikse J, et al. The origin of the medial circumflex femoral artery: a meta-analysis and proposal of a new classification system. Peer J. 2016; 4: e1726, doi: 10.7717/ peerj.1726, indexed in Pubmed: 26966661.

26. Tomaszewski KA, Vikse J, Henry BM, et al. The variable origin of the lateral circumflex femoral artery: a meta-analysis and proposal for a new classification system. Folia Morphol. 2017; 76(2): 157-167, doi: 10.5603/FM.a2016.0056, indexed in Pubmed: 27714726.

27. Uzel M, Tanyeli E, Yildirim M, et al. An anatomical study of the origins of the medial circumflex femoral artery in the Turkish population. Folia Morphol. 2008; 67(3): 226-230, indexed in Pubmed: 16988917.

28. Vazquez MT, Murillo J, Maranillo E, et al. Patterns of the circumflex femoral arteries revisited. Clin Anat. 2007; 20(2): 180-185, doi: 10.1002/ca.20336, indexed in Pubmed: 16617441.

29. Vuksanović-Božarić $A$, Abramović $M$, Vučković $L$, et al. Clinical significance of understanding lateral and medial circumflex femoral artery origin variability. Anat Sci Int. 2018; 93(4): 449-455, doi: 10.1007/s12565-018-0434-1, indexed in Pubmed: 29500659.

30. Zlotorowicz M, Czubak-Wrzosek M, Wrzosek P, et al. The origin of the medial femoral circumflex artery, lateral femoral circumflex artery and obturator artery. Surg Radiol Anat. 2018; 40(5): 515-520, doi: 10.1007/s00276-018-2012-6, indexed in Pubmed: 29651567. 